\title{
Spontaneous intracranial internal carotid artery dissection in an adolescent after heavy exercise
}

\author{
Emrah Egemen $^{1}$ [D $\cdot$ Fatih Yakar $^{1} \cdot$ Serkan Civlan ${ }^{1}$. Olcay Güngör ${ }^{2} \cdot$ Gürbüz Akçay $^{3}$
}

Received: 10 August 2021 / Accepted: 11 August 2021 / Published online: 21 August 2021

(c) The Author(s), under exclusive licence to Springer-Verlag GmbH Germany, part of Springer Nature 2021

\section{Dear Editor,}

The intracranial carotid artery dissections (ICADs) are rare, and their clinicopathological course is poorly understood [1]. Although it is frequently seen in young adults and the posterior circulation, it is more common in the anterior circulation in children [2]. Paediatric ICADs have generally been presented in small case series [3]. Delayed neurological symptoms in the paediatric population may overlook the diagnosis of ICAD [4].

We aim to present a 15 -year-old boy with ICAD following heavy exercise. The patient had a history of hypertension and was admitted to the emergency department (ED) with dysphasia and right upper limb paresis. The patient, who played volleyball at school the previous day, did body-building exercises in the evening. He stated that body-building exercises are much more intense than his regular workout routine. Three hours after exercise, his mother noticed the speech disorder and fatigue. They were admitted to ED due to weakness in the right arm within $6 \mathrm{~h}$ following the dysphasia. At admission, he was normotensive and had confusion, mild dysphasia, and $3 / 5$ right upper limb. Diffusion magnetic resonance imaging (MRI) (Fig. 1A, B) revealed multiple acute ischemic focuses on basal nuclei, frontal and parietal lobes. MR angiography (MRA) showed occlusion in the middle cerebral artery (MCA) branches (Fig. 1D), and wall irregularity at posterior communicating artery (pcomA) level in ICA (Fig. 1E) was noticed. MR venography was routine. Dissection between the ophthalmic artery and PcomA and occlusions in multiple

Emrah Egemen

egemenemrah@gmail.com

1 School of Medicine, Neurosurgery Department, Pamukkale University, Denizli, Turkey

2 School of Medicine, Division of Paediatric Neurology, Pamukkale University, Denizli, Turkey

3 School of Medicine, Division of Paediatric Intensive Care, Pamukkale University, Denizli, Turkey areas in the distal branches of the MCA were observed during cerebral digital subtraction angiography (DSA) (Fig. 1F).

The COVID-19 was negative. The antibody panel was negative without ANA (1/100 dilution). Transthoracic echocardiography, erythrocyte sedimentation rate and thrombophilia screens were normal. However, creatinine kinase was considerably higher (1045 U/L, normal < 190 U/L). Treatment was initiated with a therapeutic dose of low molecular weight heparin, but it changed with oral acetylsalicylic acid therapy following cerebral catheter angiography. He was neurologically intact at 2 months of follow-up after being discharged.

Although there is no epidemiological study on ICAD, it has been reported that it is equally affected in both genders in adults. However, it tends to be more common in males for paediatric age regardless of trauma [1]. A mural hematoma, double lumen, intimal flap and filiform appearance with areas of stenosis and dilatation are typical radiologic findings [3]. The traditional thought in dissection is the tear between the intima and the internal elastic lamina. It allows extravasation of blood to the media and subintimal or subadventitial planes [1].

The risk factors and predisposing conditions are connective tissue disorders and Moyamoya disease. While subclinical connective tissue diseases are present in the skin and arterial biopsies in patients with extracranial dissection, hypertension, hyperlipidemia and diabetes mellitus are prominent in ICAD patients [1]. Our patient had a hypertension history, but he was normotensive at admission. Furthermore, the relationship between trauma and ICAD has not been clearly explained. Akbaş et al. speculated that stretching and compression of the ICA by sudden acceleration, deceleration and rotational forces against the transverse processes or the styloid process results in dissection [5]. Everyone who was with our patient that day was questioned in detail about whether there was trauma, and it was understood that there was no trauma. However, heavy exercise may have caused dissection in our patient with the mechanism mentioned by Akbaş et al. [4].

The presentation rate with haemorrhagic events in ICAD patients is $50-60 \%$, and ischemic events are $38-70 \%$ [1]. 
Fig. 1 A Diffusion MRI revealed acute ischemic areas in the frontal and parietal lobes. B In the reconstructed MRA image, there was occlusion in MCA branches. In addition, the MRA (C) and DSA (D) revealed wall irregularity at posterior communicating artery (pcomA) level in ICA. These images verified ICAD
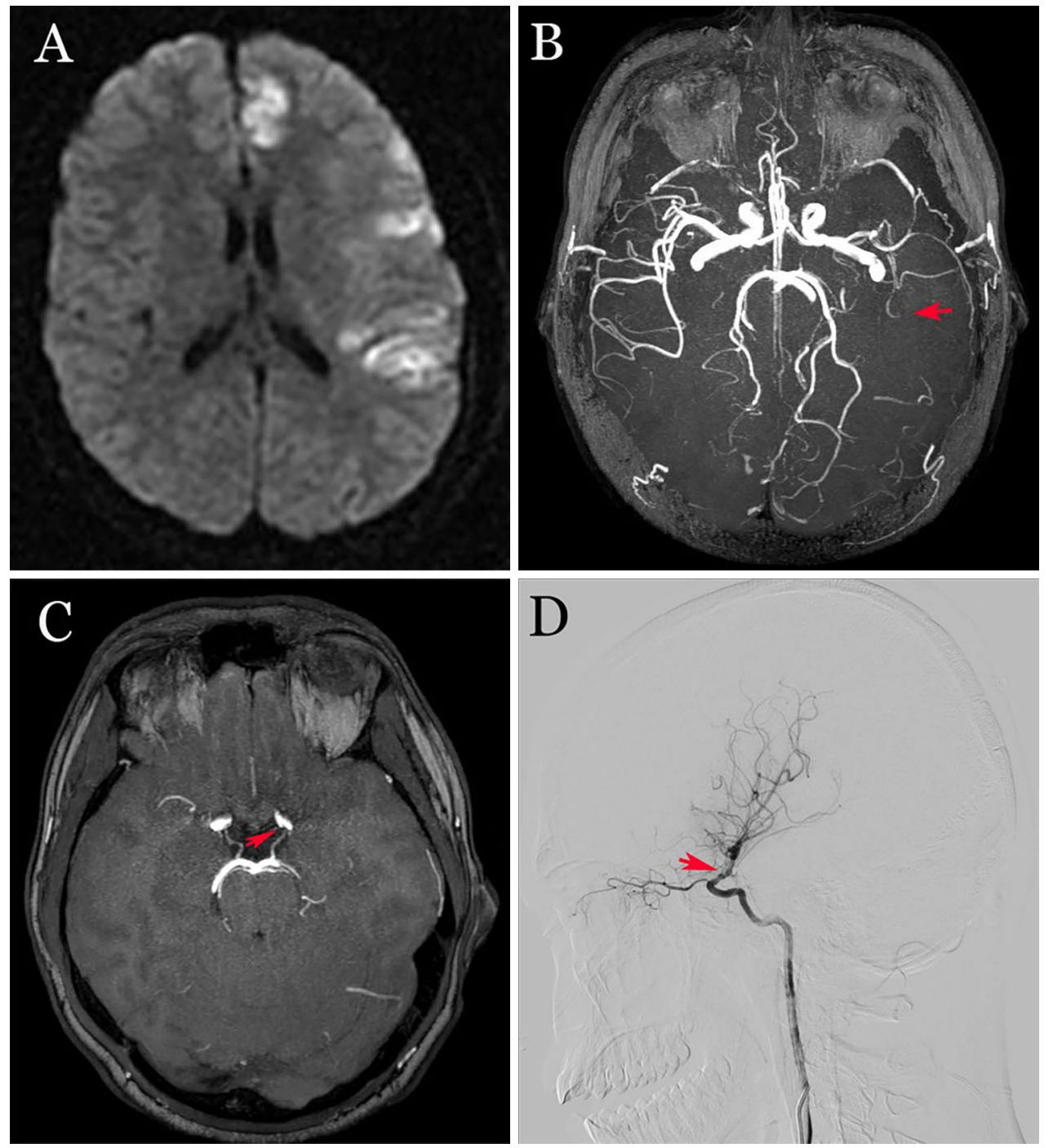

Multifocal stroke, which started with dysphasia and continued with paresis and was detected radiologically in our patient, was attributed to embolism from the thrombus to the distal branches at the dissection point. The recurrence rate is $2-38 \%$ in patients presenting with ischemic events in the adult population. However, no actual rate can be given in the paediatric age group [1].

There is no current guideline for ICAD management. In medical treatment, anticoagulant or antiplatelet therapy is recommended to prevent recurrent strokes [1]. Since anticoagulation may lead to subarachnoid haemorrhage in ICAD, it is recommended to prefer antithrombotic therapy (Class III recommendations). Therefore, we administered low molecular weight heparin, but after the diagnosis was confirmed by catheter angiography, it was replaced with antiplatelet (aspirin) treatment. There is no sufficient data on intravenous thrombolysis. There are no contraindications for endovascular recanalization in ICADs resulting in large vessel occlusion. Endovascular stenting or surgical bypass can be planned for patients who develop recurrent stroke despite medical treatment [5].

Although there is no current guideline for ICAD treatment and follow-up, early recognition of stroke in paediatric patients will give the chance of endovascular treatment.

Acknowledgements The authors are thankful to Kenichi Sato, MD (Tohoku Medical and Pharmaceutical University, Department of Neurosurgery) for his assistance in evaluating the radiological images.

\section{Availability of data and material N/A}

Code availability N/A

\section{Declarations}

Conflict of interest On behalf of all authors, the corresponding author states that there is no conflict of interest. 


\section{References}

1. Bond KM, Krings T, Lanzino G, Brinjikji W (2021) Intracranial dissections: a pictorial review of pathophysiology, imaging features, and natural history. J Neuroradiol 48(3):176-188. https:// doi.org/10.1016/j.neurad.2020.03.007

2. Fullerton HJ, Johnston SC, Smith WS (2001) Arterial dissection and stroke in children. Neurology 57(7):1155-1160. https://doi. org/10.1212/WNL.57.7.1155

3. Rafay MF, Armstrong D, Deveber G, Domi T, Chan A, MacGregor DL (2006) Craniocervical arterial dissection in children: clinical and radiographic presentation and outcome. J Child Neurol 21(1):8-16. https://doi.org/10.1177/ 08830738060210010101
4. Akbaş Y, Arhan E, Serdaroglu A, Nazlıel B (2016) Intracranial internal carotid artery dissection following waterslide use: the first case report. Childs Nerv Syst 32(3):411-413. https://doi.org/10.1007/ s00381-016-3024-3

5. Roach ES, Golomb MR, Adams R, Biller J, Daniels S, Deveber G, Ferriero D, Jones BV, Kirkham FJ, Scott RM, Smith ER (2008) Management of stroke in infants and children: a scientific statement from a Special Writing Group of the American Heart Association Stroke Council and the Council on Cardiovascular Disease in the Young. Stroke 39(9):2644-2691. https://doi.org/10.1161/ STROKEAHA.108.189696

Publisher's Note Springer Nature remains neutral with regard to jurisdictional claims in published maps and institutional affiliations. 